\title{
OBITUARIO
}

\section{Patricia Elena Emilia Madrid}

\section{$(17 / 01 / 1954-20 / 04 / 2021)$}

\author{
Por Gustavo Martínez
}

Agradezco a Intersecciones en Antropología la invitación para escribir estas líneas sobre mi amiga y colega Patricia Madrid. No solo lo agradezco porque me da un lugar para mencionar lo que ella significó para muchos de nosotros y contar a su vez su trayectoria académica, sino que es una gran oportunidad para realizar una suerte de catarsis ante la imposibilidad de despedirla como hubiésemos querido debido a la pandemia que atravesamos. Naturalmente, no podré despojarme de mi visión de Patricia y seré autorreferencial, aunque tengo serios indicios para sostener que mucho de lo que contaré son opiniones compartidas por la mayoría de la gente que bien la conoció. Conocí a Pato en marzo de 1987, en mi primer año de la carrera de Antropología en la Facultad de Ciencias Naturales y Museo (UNLP). Durante el curso de ingreso y recientemente comenzadas las cursadas, nos enteramos de que había altas chances de participar tempranamente de excavaciones arqueológicas, lo cual en ese entonces ya nos sacaba el sueño. Un ayudante de la cátedra de Geología me sugirió acercarme al laboratorio de Gustavo Politis, en el subsuelo del museo en ese entonces. Luego de una cursada, notablemente nervioso, golpeé la puerta del laboratorio. Salió Patricia, me atendió muy amablemente y escuchó con atención mis intenciones de ir al campo y, si era posible, hacer algún trabajo de laboratorio. Me comentó que en ese momento no tenían previstas salidas al campo, pero que pasara cada tanto por si surgía algo. Palabras más, palabras menos, con el tiempo me confesó "Pensé que eras un primo de Politis". Ese primer contacto no fue en vano y en mayo de ese año, Patricia nos invitó a excavar el sitio Claverizo (partido de Tornquist). La experiencia fue maravillosa, a pesar del intenso frío que pasaba las paredes de la casiIla rural de chapa donde dormíamos. Si algo faltaba para terminar de afianzar nuestras vocaciones, Patricia, con su energía y pasión, nos terminó de convencer. Como si este rito iniciático fuese poco, ni bien regresamos a La Plata nos invitó a concurrir los viernes a la tarde al laboratorio, donde comenzó a enseñarnos a analizar artefactos líticos. El hecho de que ese día hiciéramos arqueología "en serio" era sumamente excitante y nos motivaba a darles duro a las cursadas porque hacia el final de la semana teníamos nuestro premio. Así, en el pequeño laboratorio, en una de las improvisadas mesadas, Pato desplegaba la fotocopia del "Ensayo para una clasificación...", de Carlos Aschero y, cuarcitas en mano, comenzaba a enseñarnos qué mirar, cómo mirar, qué era un núcleo, qué era una lasca. En la medida en que nos fuimos conociendo, los viernes a la tarde se fueron haciendo cada vez más largos, ya que desde el museo nos íbamos directo al departamento de Patricia, donde nos esperaba una guitarra y, como ella decía en ese entonces, un "fratello", que acompañábamos con una picada, empanadas o, si estábamos inspirados, guiso de lentejas. Pato ejecutaba una deliciosa versión de "La Pomeña" en guitarra y voz. Su departamento estaba Ileno de

Gustavo Martínez. UE-INCUAPA-CONICET. FACSO-UNICEN. Av. Del Valle 5737 (CP 7400), Olavarría, Argentina. E-mail: gmartine@soc.unicen.edu.ar 
literatura y música. Claro, ahí entendimos que su sensibilidad por el arte había hecho que, antes de emprender su aventura arqueológica, estudiara tres años de Licenciatura en Pintura en la Facultad de Bellas Artes (UNLP).

Patricia se graduó como licenciada en Antropología en la Facultad de Ciencias Naturales y Museo (UNLP) en el año 1985. Desarrolló una profusa y diversa actividad en docencia, investigación, gestión y extensión. En docencia transitó todos los cargos, desde ayudante diplomada hasta profesora titular por concurso, actividad respecto de la cual haré una breve mención, dada la larga trayectoria que posee. En la FCNyM, fue docente en las asignaturas "Arqueología Americana II" y "Métodos y Técnicas de la Investigación Arqueológica". En materia de gestión se desempeñó, entre muchos otros cargos, como secretaria de la Comisión Directiva del Centro de Graduados y Miembro Titular del Consejo Consultivo Departamental de Antropología. Comenzó su actividad en la Facultad de Ciencias Sociales (UNICEN) en 1990, dictando clases en las asignaturas "Teoría Arqueológica", "Arqueología de Grupos Cazadores-Recolectores Americanos", "Arqueología Argentina II" y "Arqueología de Grupos Agricultores Americanos". Su labor de gestión en esta unidad académica fue muy prolífica: fue consejera académica (seis periodos) y consejera superior (dos periodos). Desde 1998 fue miembro titular de la Junta Departamental de Arqueología y se desempeñó como directora del Departamento de Arqueología entre 2005 y 2016. Entre los muchos logros de su gestión se destaca la modificación del Plan de Estudios de la Licenciatura en Antropología con orientación Arqueología (2014). La dimensión de esta enorme tarea de Patricia se pone de relevancia si se considera que siempre fue una docente-investigadora "viajera". Es notable, entonces, la dedicación y el profesionalismo que Patricia invirtió durante los días de estadía en Olavarría al servicio del Departamento de Arqueología y de la FACSO.

En lo referido a la investigación científica, era dueña de una profunda curiosidad que la llevaba a interesarse por una variedad de temáticas. Si bien su actividad de investigación se basó principalmente en el estudio de las sociedades cazadoras-recolectoras pampeanas, Patricia siempre mencionaba que otra muy buena opción para ella hubiese sido hacer arqueología del noroeste argentino. Siempre recordaba sus trabajos de campo iniciales en esta región y se preguntaba por qué su trayectoria de investigación no se había desarrollado allí. El caso es que con la pasión y el compromiso que la caracterizaban abrazó fuertemente la arqueología pampeana. Sus trabajos iniciales fueron a principios de la década de 1980 cuando, aun siendo alumna, colaboró con el Dr. Gustavo Politis en la investigación de diversos sitios arqueológicos, entre ellos, La Toma. En 1983 tuvo una pasantía de la Dirección de Recursos Naturales y Ecología del Ministerio de Asuntos Agrarios de la Provincia de Buenos Aires para realizar investigaciones arqueológicas en el Parque Provincial E. Tornquist (Sierra de la Ventana). Posteriormente, desde 1986, obtuvo también becas diversas del CONICET.

Entre los temas que abordó, Patricia fue una especialista en el estudio de la tecnología cerámica y lítica. En el caso de la primera, tomó cursos con referentes en el tema (D. Peacock) durante su estadía en el Departamento de Arqueología de la Universidad de Southampton (1992). Fue una pionera en el estudio de pastas y petrología cerámicas en la región. Realizó un detallado estudio basado en cortes delgados y análisis microscópicos en varios sitios del Holoceno tardío de la región y definió grupos cerámicos (Madrid, 1997). Desde luego, también llevó a cabo estudios tecnomorfológicos de esta cultura material, tanto en la Región Pampeana como en la transición Pampeano-Patagónica oriental (Madrid y Politis, 1991; Borges Vaz, Martínez y Madrid, 2016). Siempre fue una referente en estudios de tecnología lítica, y contribuyó al análisis e interpretación de los conjuntos arqueológicos de diversos sitios (e.g., Tres Reyes, La Toma, Quequén Salado 1, entre muchos otros) (Madrid y Salemme, 1991; Madrid y Politis, 1991; Madrid et al., 1991, 2002; March et al., 2006). Como parte del estudio sistemático y de larga data de sitios arqueológicos pampeanos que fueron fundamentales para la construcción de modelos de variada índole en la región (e.g., Tres Reyes, La Toma, Arroyo Seco), realizó contribuciones en estudios zooarqueológicos, tafonómicos y de procesos de formación de sitios (Politis y Madrid, 1988; Politis, Madrid y Barrientos, 1992; Salemme y Madrid, 2007), así como publicaciones referidas a reconstrucciones paleoambientales (Madrid y Politis, 1991; Salemme y Madrid, 2007). Otros trabajos tuvieron como objetivo analizar la estructura del registro arqueológico y bioarqueológico, las modalidades de entierro de sitios particulares (e.g., Tres Reyes) y establecer comparaciones con otros sitios de la región (Madrid y Barrientos, 2000). Patricia también participó en el marco de proyectos internacionales con una 
aproximación microrregional, como el caso de la cuenca del río Quequén Salado, trabajo que contribuyó al hallazgo y estudio de una quincena de sitios arqueológicos. Patricia no solo aportó a la generación de conocimiento sobre diversas líneas de análisis (e.g., lítico) (March et al., 1999, 2006; Madrid, et al., 2002), sino que fue codirectora de este proyecto de cooperación internacional argentino-francés (FACSO, UNICEN y Universidad de Rennes 1, Francia - 1999-2003- SECYT-ECOSud).

El estudio de las estructuras de piedra y el arte rupestre no estuvieron ausentes en sus investigaciones. Un caso de estudio en el que ambas manifestaciones se registran en forma conjunta es el de la localidad arqueológica Sierras de Curicó, proyecto en el cual Patricia tuvo un rol protagónico. En ambos cerros que componen la localidad se registraron estructuras de pirca, montículo de piedras, un alero con material en estratigrafía y sectores con pinturas rupestres (e.g., grecas) asignados a sociedades cazadoras-recolectoras de los últimos ca. 500 años AP (Madrid, Politis y Poire, 2000; Pedrotta, Madrid y Politis, 2009). Posteriormente, a los resultados arqueológicos obtenidos de esta localidad se anexó la evaluación del estado de preservación de algunas manifestaciones (e.g., pinturas rupestres, construcciones de piedra), de su valor científico-académico y de las condiciones ambientales, sociales y económicas ligadas a la protección y puesta en valor. Así, se efectuaron recomendaciones respecto del uso de la localidad como parte de circuitos de turismo cultural, para el que se propuso un modelo de gestión patrimonial y plan de manejo (Pedrotta et al., 2009; Madrid, Pedrotta e Istillarte, 2010). Con anterioridad, Patricia ya se había interesado por el arte rupestre de Sierra de la Ventana, donde investigó paneles registrados en cuevas y aleros (Madrid y Oliva, 1994).

También realizó contribuciones que sintetizan el estado del arte de la arqueología pampeana, en las que se analizan cuáles fueron los problemas centrales objeto de análisis y cuáles los ejes espaciales y cronológicos bajo los que se enmarcó la discusión de dichos problemas. En este sentido, publicaciones como las de Politis y Madrid (2001) son hoy trabajos clásicos de consulta obligada para la región.

Sin embargo, las contribuciones de Patricia fueron más allá de la arqueología de sociedades prehispánicas. En línea con la temática de estudio vinculada a las estructuras de piedra se destaca el intenso relevamiento realizado en las sierras de
Pillahuincó (partidos de Coronel Pringles y Coronel Suárez), donde relevó una veintena de recintos con paredes de piedra y piedras paradas. Así, a la documentación histórica existente le añadió información arqueológica que la llevó a sugerir el origen indígena de la mayoría de los sitios estudiados y su pertenencia a un sistema de asentamiento vinculado a un circuito de mantenimiento y traslado de ganado (Madrid, 1991). Además, fue parte de un proyecto en arqueología histórica a través de la investigación del Fuerte Blanca Grande (partido de Olavarría). Los objetivos de este fueron el estudio de los aspectos arquitectónicos y de construcción, entender el uso del espacio dentro y fuera del fuerte, conocer aspectos de su reocupación durante su funcionamiento y aportar, a partir del estudio de la cultura material, a la comprensión de los modos de vida fronterizos durante parte del siglo XIX (Goñi y Madrid, 1998). Producto de este proyecto se generaron tesis de licenciatura con las cuales se graduaron estudiantes de la FACSO-UNICEN. El rol de Patricia como codirectora de este proyecto fue de suma importancia (1992-1997; Proyecto UNCPBA).

Proyectos referidos a arqueología, educación y extensión no estuvieron ajenos de su agenda de trabajo. Junto con un nutrido grupo de becarios e investigadores de la FCNyM (UNLP), de la FACSO e INCUAPA (UNICEN), promovió el desarrollo de una diversidad de tareas de extensión en localidades de las provincias de Buenos Aires, Entre Ríos y Río Negro. Talleres, charlas-debate, conferencias, pósters, preparación de exhibiciones en museos, producción de artículos informativos, ferias de ciencias y muestras fotográficas fueron organizados en el ámbito de museos regionales, escuelas primarias y secundarias e instituciones con asistencia de público en general. Estos proyectos de comunicación pública de la arqueología -cuidadosamente planificados y diseñados- contaron con la participación de especialistas en educación y comunicación (Bonomo, et al., 2010; Di Prado, et al., 2012; Conforti et al., 2013). Durante un periodo, Patricia estuvo a cargo de la dirección de uno de ellos (proyecto financiado por la Comisión de Extensión del Honorable Consejo Superior de la UNLP) y fue coordinadora en otros tramos de su ejecución. Estas tareas de extensión, que en esta etapa se observan más formales y organizadas, ya eran de interés para Patricia desde la década de 1980, cuando junto con otros colegas organizó y participó de charlas, encuentros regionales y de directores de museos en diversos partidos del sur bonaerense. En este sentido, fue 
parte de la comisión organizadora de las Primeras Jornadas Arqueológicas Regionales, Ilevadas a cabo en 1989 en Pigüé (Centro de Registro del Patrimonio Arqueológico y Paleontológico, 1991).

Una de sus últimas contribuciones, en tanto trabajo de campo y análisis, la realizó en Patagonia meridional a partir del estudio de estructuras de piedra (parapetos) y de las tácticas de caza empleadas por cazadores-recolectores para la obtención de guanacos (Belardi et al., 2017). En el sur de la provincia de Santa Cruz participó de estudios de impacto ambiental en colaboración con el equipo de arqueología de la Universidad Nacional de la Patagonia Austral.

Además de lo que ya he mencionado, Patricia formó parte de numerosos proyectos de investigación vinculados al Laboratorio 3 de la Facultad de Ciencias Naturales y Museo de la UNLP, al Departamento de Arqueología e INCUAPA-FACSOUNICEN, financiados por distintas instituciones (PIPCONICET, ANPCYT, UNICEN), y dirigió y codirigió proyectos relacionados con temas de educación e identidad local (PICTO). Formalmente, ha dirigido a estudiantes y graduados en el marco de pasantías y becas CONICET, FCNyM-UNLP, CIN, así como tesis de grado. Participó de la organización de diversos eventos científicos (e.g., CARPA) y como coeditora de numerosas publicaciones.

El contenido de los párrafos precedentes muestra la diversidad de temas arqueológicos en los cuales Patricia trabajó y cómo se involucró en distintos proyectos, a los que aportó siempre su seriedad, rigurosidad y enorme capacidad de trabajo en la investigación científica. Estas características aplican también a su actividad de gestión que, además de muy intensa, siempre fue de un enorme compromiso para con las instituciones a las que representaba.

Hay un aspecto de Patricia que a mi entender es uno de los más destacados de su personalidad: el compromiso en la formación de recursos humanos. Como he mencionado al comienzo, ella fue para algunos de nosotros la persona que muy tempranamente en nuestras carreras nos introdujo en el trabajo de campo y laboratorio y nos mostró que era posible ser un arqueólogo, que valía la pena apostar a esta labor. En mi caso, al menos, el entusiasmo que me generó esa experiencia temprana impactó positivamente en la forma en que seguí adelante en la profesión. Pero presumo que este no es un hecho aislado y que ha sido el caso de varias generaciones de estudiantes, tanto en La Plata como en Olavarría. Una cualidad no menor era el desinterés con el que ella invertía generosamente su tiempo y su conocimiento en la formación de los estudiantes y graduados jóvenes. Para ella era irrelevante si la formación de esa persona se enmarcaba formalmente dentro de una beca que aportara a sus antecedentes profesionales. El entusiasmo y dedicación con que formaba a los estudiantes eran exactamente los mismos en cualquier circunstancia. Además, poseedora de una calidad humana enorme, su compañía era siempre grata y generaba una atmósfera que potenciaba aún más las jornadas de aprendizaje.

En Olavarría, Patricia tuvo su lugar de trabajo en un box ubicado en la biblioteca del INCUAPA. En cada uno de sus viajes, y sobre todo mientras fue la directora del Departamento de Arqueología, transformaba este espacio en una suerte de "consultorio" por el que desfilábamos alumnos, docentes, becarios, investigadores, personal técnico y administrativo. En estas circunstancias se repetía el mismo ritual que, como mencioné arriba, constituía uno de los mayores valores que Patricia tenía: su enorme generosidad con su tiempo y su conocimiento. Durante el incesante desfile que duraba los dos días de su estadía quincenal en Olavarría, se mezclaban consultas e inquietudes de toda índole, no solo científico-académicas, que Patricia escuchaba muy atentamente brindando siempre su opinión sobre el tema que se tratase.

Sin dudas, Patricia fue mucho más de lo que aquí he escrito y debo reconocer que cualquier valoración que realice estará sesgada por la amistad que mantuvimos desde que nos conocimos. Lamentablemente, esta pandemia hizo que no pudiéramos acompañarla todo lo que hubiésemos querido, todo lo que ella merecía, sobre todo en sus últimos y difíciles meses. Es por esto que su ausencia parece todavía una ilusión, y su muerte, algo que nunca sucedió. A riesgo de demorar el duelo y sus consecuencias, aprovechemos para cruzárnosla en los pasillos de la Facultad, charlar divertidamente con ella y verla reírse estridentemente, sentada, con sus piernas cruzadas, sobre la mesa de la biblioteca del INCUAPA.

\section{Agradecimientos}

Gracias al Comité Editorial de Intersecciones en Antropología por darme la oportunidad de escribir estas líneas sobre Patricia. Agradezco a F. Carballo 
Marina, L. Miotti, M. Salemme, M. Bonomo, G. Politis y $M$. Gutiérrez por brindarme información científico-académica, por la lectura del manuscrito y por refrescar mi memoria sobre hechos que Patricia me contó alguna vez. A A. P. Alcaráz, por su ayuda con el manuscrito.

\section{REFERENCIAS CITADAS}

Belardi, J. B., Carballo Marina, F., Madrid, P., Barrientos,

G., y Campan, P. (2017). Late Holocene guanaco hunting grounds in southern Patagonia: blinds, tactics and differential landscape use. Antiquity, 91(357), 718-

731. doi: https://doi.org/10.15184/aqy.2017.20

Bonomo, M., Prates, L., Madrid, P., Di Prado V., León, C., Angrizani, R.,... Bagaloni V. (2010). Arqueología. Conocer el pasado a través de los objetos. Museo, 3(24), 16-28. http://www.memoria.fahce.unlp.edu.ar/ art_revistas/pr.10005/pr.10005.pdf

Borges Vaz, E., Martínez G. y Madrid P. (2016). Análisis tecno-morfológicos y tendencias cronológicas del conjunto cerámico del sitio Loma Ruíz 1 (transición pampeano-patagónica oriental). Aportes para Pampa y Norpatagonia. Intersecciones en Antropología, 17, 269-280.

Centro de Registro del Patrimonio Arqueológico y Paleontológico (1991). Boletín del Centro de Registro del Patrimonio Arqueológico y Paleontológico.

Dirección Museos, Monumentos y Sitios Históricos.

Conforti, M. E., Madrid, P., Bonomo, M. y Prates, L. (2013). The Public Communication of Science and Non-Formal Education Within the Framework of Public Argentine Archaeology: The Case of Archaeology Workshops. Public Archaeology, 12(1), 27-47.

Di Prado, V., Matarrese, A., Bagaloni, V., Bonomo, M., Prates, L., Angrizani, R. y Madrid, P. (2012). Arqueología, Educación y Museos. Encuentro entre investigadores y comunidades locales. Experiencias en Extensión, 1-6. http://sedici.unlp.edu.ar/ handle/10915/95584

Goñi, R. y Madrid, P. (1998). Arqueología sin hornear: sitios arqueológicos de momentos históricos y el Fuerte Blanca Grande. Intersecciones en Antropología, 2, 39-50.

Madrid, P. (1991). Estudio Arqueológico de los Sitios con Estructuras de Piedra en las Sierras de Pillahuincó, Provincia de Buenos Aires. Revista del Museo de Historia Natural de San Rafael, Mendoza, 11(3), 129-156.
Madrid, P. (1997). Análisis petrológico y alfarería pampeana. En M. Berón y G. Politis (Eds.), La arqueología de la región pampeana en la década de los '90 (pp. 61-70). Museo de Historia Natural de San Rafael, Mendoza, INCUAPA, FCNyM, UNICEN.

Madrid, P. y Barrientos, G. (2000). La estructura del registro arqueológico del sitio Laguna Tres Reyes 1 (Provincia de Buenos Aires): nuevos datos para la interpretación del poblamiento humano del sudeste de la Región Pampeana a inicios del Holoceno tardío. Relaciones de la Sociedad Argentina de Antropología, 25, 179-206.

Madrid, P. y Oliva, F. (1994). Análisis preliminar de las representaciones rupestres presentes en cuatro sitios del Sistema de Ventania, Pcia. de Buenos Aires. Revista del Museo de La Plata, 9, 199-223.

Madrid, P., Pedrotta, V. e Istillarte, A. (2010). Patrimonio Arqueológico, Preservación y Turismo. El Caso De Las Sierras De Curicó (Sistema de Tandilia, Región Pampeana, Argentina). En F. Carballo Marina y L. Manzi (Eds.), Informe científico técnico. Secretaría de Ciencia y Tecnología de la Universidad Nacional de la Patagonia Austral, Secretaría de Investigación y Postgrado de la Unidad Académica Río Gallegos, Universidad Nacional de la Patagonia Austral. ICTUNPA-18-2010. Recuperado de http://168.226.35.7/ secyt/ict/

Madrid, P. y Politis, G. (1991). Estudios arqueológicos y paleoambientales en la Región Pampeana: un enfoque multidisciplinario del sitio La Toma. Actas XI Congreso Nacional de Arqueología Chilena, 1, 131-153.

Madrid, P. y Salemme, M. (1991). La ocupación tardía del sitio 1 de la Laguna Tres Reyes, Pdo. de Adolfo Gonzáles Chaves (Pcia. de Buenos Aires). Boletín del Centro, 3, 165-179.

Madrid, P., Politis, G. y Poire, D. (2000). Pinturas rupestres y estructuras de piedra en las Sierras de Curicó (Extremo noroccidental de Tandilia, Región Pampeana. Intersecciones en Antropología, 1(1), 35-53.

Madrid, P., Politis, G., Leipus, M. y Landini, C. (1991). Estado actual de las investigaciones en el Sitio 1 de la Laguna Tres Reyes: análisis lítico tecno-morfológico y procesos de formación del sitio. Boletín del Centro, 2, 112-122.

Madrid, P., Politis, G., March, R. y Bonomo, M. (2002). Arqueología microrregional en el Sudeste de la Región Pampeana: el Curso del río Quequén Salado. Relaciones de la Sociedad Argentina de Antropología, 27, 327-355. 
March, R., Laporte, L., Karlin, C., Joly, D., Politis, G., Madrid, P. y Bonomo, M. (1999). Un nouveau projet international de I'UMR: Evolution et Adaptation Humaine autour de la Region des Plaines et Lacs de Fleuve Quequén Salado, Buenos Aires (Argentina). Journes Prehistoriques de Bretagna. Université de Rennes, 1, 43-48.

March, R. J., Rodriguez Loredo, C., Madrid, P., Politis, G., Bonomo, M., Joly, D.,...Karlin, C. (2006). Évolution et adaptation humaine autour de la région des plaines et lacs du fleuve Quequén Salado. Pcia. de Buenos Aires (Argentine): premiers pas d'une archéologie régionale programmé: la prospection. En S. Purin (Presidencia), Acts of the XIVth UISPP Congress, University of Liège, Belgium. Section 17: Préhistoire de I'Amérique (pp. 211-231). BAR International Series 1524, Archaeopress.

Pedrotta, V., Madrid, P. y Politis, G. (2009). Pinturas, Pircas y Aleros en las Sierras de Curicó (Partido de Olavarría). En J. L. Prado y M. L. Endere (Eds.), Patrimonio natural y cultural de la región comprendida entre los partidos de Azul, Olavarría y Tandil (pp. 187-206). Universidad Nacional del Centro de la Provincia de Buenos Aires.
Politis, G. y Madrid, P. (1988). Un hueso duro de roer: análisis preliminar de la tafonomía del sitio Laguna Tres Reyes 1 (Pdo. de Adolfo González Chaves, Pcia. de Buenos Aires). En N. Ratto y A. Haber (Eds.), De procesos, contextos y otros huesos (pp. 29-44). Instituto de Ciencias Antropológicas, Facultad de Filosofía y Letras. UBA.

Politis, G. y Madrid, P. (2001). Arqueología Pampeana: Estado actual y perspectivas. En E. Berberian y A. Nielsen (Eds.), Historia argentina prehispánica, Tomo II (pp. 737-814). Brujas.

Politis, G., Madrid P. y Barrientos, G. (1992). Informe de la campaña 1992 al sitio Arroyo Seco 2 (Pdo. De Tres Arroyos, Pcia de Buenos Aires, Argentina). Palimpsesto, 1, 80-83.

Salemme, M., y Madrid, P. (2007). The Archaeofaunas from Laguna Tres Reyes 1 Site: taxonomic richness and abundance during the beginning of the Late Holocene in the south-east Pampean Region (Argentina). En M. Gutiérrez, L. Miotti, G. Barrientos, G. Mengoni Goñalons y M. Salemme (Eds.), Taphonomy and Zooarchaeology in Argentina (pp. 121-143). BAR International Series 1601, Archaeopress. 